\title{
Sur la relation entre la teneur en bêta-carotène et la couleur des beurres français
}

\author{
par \\ J. CASALIS*, Y. CHARDON*, F. M. LUQUET***, P. MAINGUY** \\ et M. YVER** \\ avec la collaboration technique de Ch. BONDUELLE***
}

\section{Introduction}

La couleur est incontestablement un des critères essentiels de choix du consommateur en ce qui concerne les denrées alimentaires. Pour ce qui est du beurre, le consommateur français en général, réclame une coloration jaune assez prononcée. Or, suivant les saisons, suivant la race du bétail laitier, suivant les régimes alimentaires des animaux, la couleur naturelle des beurres français varie dans des proportions considérables. Le but de la présente étude était de mettre au point une méthode scientifique d'appréciation de la couleur du beurre et de rechercher les moyens précis de la normaliser, en utilisant comme colorant le $\beta$-carotène dont on sait qu'il ne fait courir aucun risque à la santé du consommateur et qu'il est l'un des principaux pigments naturels du lait.

\section{I. - Rappels de colorimétrie}

A) LA NOTION DE COULEUR

Lorsque la notion de couleur intervient dans le langage courant, on exprime, par un mot ou une phrase, une sensation physiologique déterminée, qui est la résultante de plusieurs phénomènes physiques perçus simultanément.

Préciser une couleur revient à utiliser généralement plusieurs qualificatifs permettant de parfaire l'information que l'on désire donner.

* Laboratoire du Lait - ENSIA, Douai.

** F. Hoffmann-La Roche, Paris.

*** LRIL-DSV, Douai. 
L'analyse de cette information montre que l'on est toujours amené à distinguer :

$1^{\circ}$ La teinte, permettant d'opérer un choix élémentaire dans la gamme des couleurs, par exemple, vert, jaune, bleu ;

$2^{\circ}$ La saturation qui exprime la proportion du mélange de cette teinte avec le blanc. Un jaune peut être franc ou pâle. La teinte et la saturation définissent la chromaticité ;

30 L'éclat permettant de différencier deux couleurs de même teinte et de même saturation. Nous parlons d'un jaune lumineux ou d'un jaune terne.

$$
\text { Couleur }\left\{\begin{array}{c}
\text { teinte } \\
+ \\
\text { saturation } \\
+ \\
\text { éclat }
\end{array}\right\} \text { chromaticité }
$$

fig. 1

La notion de couleur est à la fois complexe et relative : complexe parce que les phénomènes physiques mis en jeu sont nombreux ; relative en raison des possibilités de variation du récepteur nerveux que constitue l'œil humain.

De nombreux appareils de physique ne sont sensibles qu'à une seule caractéristique de la lumière, généralement son énergie. Quelle que soit la nature de la lumière étudiée, ces appareils ne réagissent qu'en fonction de la quantité d'énergie qu'ils reçoivent et mesurent; on dit qu'ils sont univariants.

L'œil, par contre, est trivariant, c'est-à-dire qu'en plus du flux lumineux, deux variables suffisent pour caractériser l'infinie variété des compositions spectrales. En d'autres termes, avec trois couleurs dites couleurs primaires, par exemple le bleu, le vert et le rouge, l'œil est capable de reconstituer toutes les couleurs.

\section{B) Appréciation et mesure de la couleur}

D'un point de vue général, l'estimation d'une couleur se fondant sur les trois caractéristiques : la teinte, la saturation et l'éclat, est une opération toujours difficile, liée aux imprécisions de l'observateur et à celles du vocabulaire utilisé pour essayer de traduire les impressions reçues.

Si l'on veut définir une couleur à l'aide de moyens techniques quelconques plus élaborés que l'appréciation empirique usuelle, il convient de définir tout d'abord le degré de précision que l'on désire atteindre dans ses évaluations.

Pour les observations usuelles et l'appréciation commerciale, nous avons besoin de méthodes simples et commodes d'emploi dont la précision doit être ajustée aux besoins réels. 
Pour les travaux de recherche, il est indispensable de disposer de mesures rigoureuses.

C) Méthodes de mesure de la couleur

\section{1) Les méthodes subjectives de mesure de la couleur}

Les méthodes subjectives sont fondées sur la comparaison avec une série d'étalons.

Il est indispensable que ces étalons soient invariables de façon à assurer la fidélité des mesures.

Citons par exemple les teintomètres du type Lovibond, les systèmes à disques du type Munsell, les échelles pour un produit (échelle Roche pour les œufs).

\section{2) Les méthodes indirectes d'appréciation de la couleur}

Les méthodes indirectes utilisent un moyen de mesure photométrique et peuvent comporter un traitement plus ou moins complexe de l'échantillon à examiner, depuis l'extraction simple de la fraction colorée jusqu'à la séparation, la caractérisation et le dosage des divers pigments du produit.

3) Les méthodes objectives de mesure de la couleur : Le système des coordonnées XYZ de la C.I.E.

De nombreux chercheurs se sont attachés à mettre au point des méthodes générales qualitatives et quantitatives d'appréciation des couleurs, fondées sur la trichromie.

La Commission Internationale de l'Eclairage (C.I.E.), en 1931, a retenu le système XYZ permettant, à partir de mesures physiques appropriées, de définir toutes les couleurs possibles au moyen de trois coordonnées.

C'est cette méthode qu'il convient d'employer pour aboutir à des résultats cohérents.

La théorie de la trichromie est trop complexe pour être exposée complètement en quelques lignes ; cependant, en prenant quelques libertés avec la rigueur des principes, nous voudrions en faire comprendre les bases.

Nous avons vu qu'en additionnant, selon des proportions variables, trois radiations convenablement choisies, dites primaires, il était possible de reproduire toutes les couleurs.

Le problème pratique qui se pose est de trouver une représentation graphique simple de ce phénomène.

Pour ce faire, on a choisi arbitrairement trois radiations primaires (rouge, vert, bleu) que l'on a figurées par les trois sommets R (red), G (green), B (blue) d'un triangle appelé triangle des couleurs (fig. 2). 


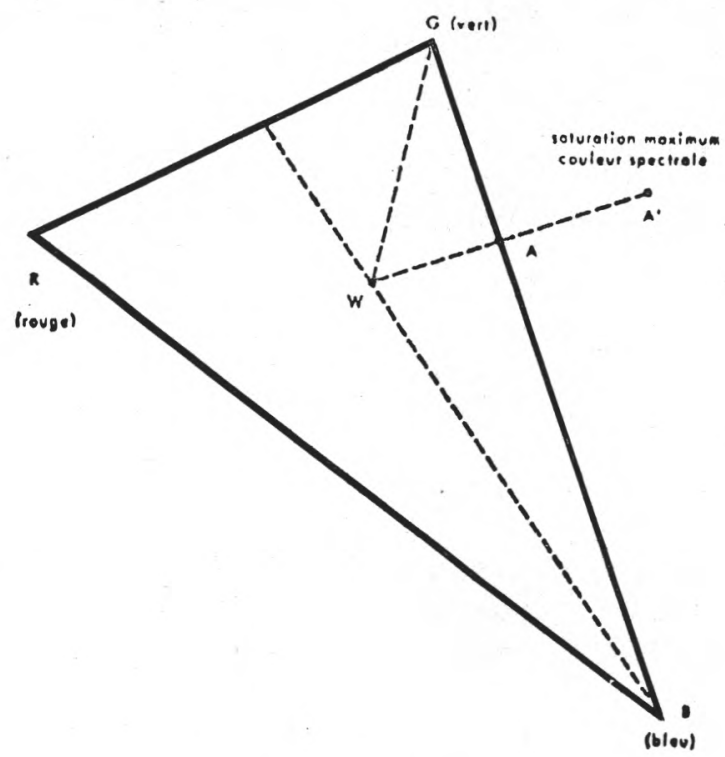

Triengle des couleurs R.G. D.

fig. 2

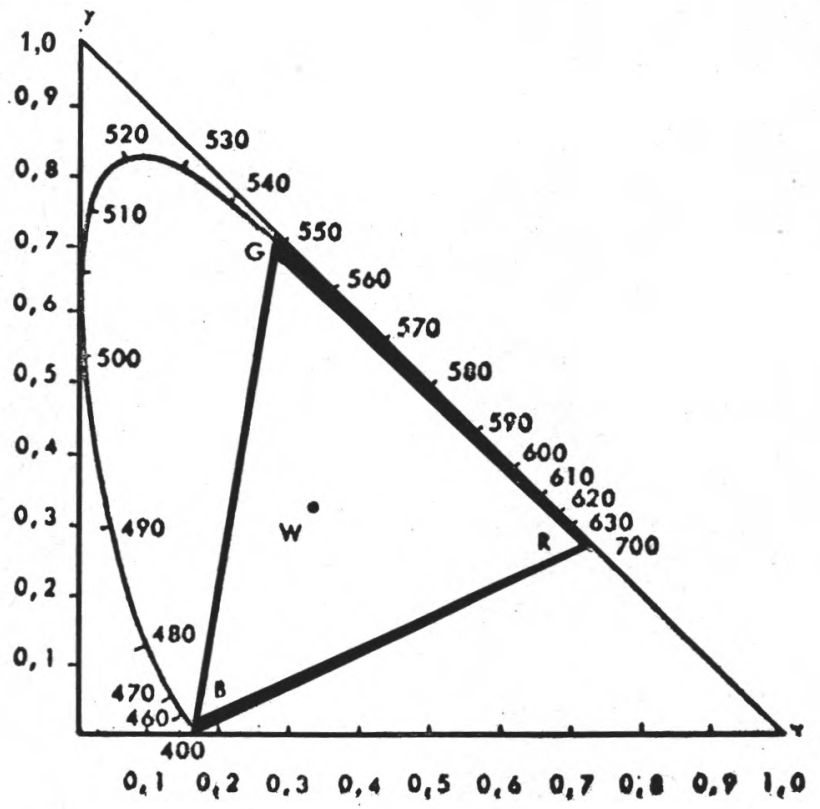

fig. 3 
Une addition judicieuse $\mathrm{du}$ bleu, du vert et du rouge donne le blanc qui se situe au centre $\mathrm{W}$ et les additions en proportions variables de $\mathrm{R}, \mathrm{G}$ et $\mathrm{B}$ peuvent être figurées par un point à l'intérieur du triangle.

Mais si, à partir du point $\mathrm{W}$, on veut obtenir, pour une teinte donnée différente de $\mathrm{R}, \mathrm{G}, \mathrm{B}$ et déterminée par une longueur d'onde quelconque, toutes les saturations expérimentalement possibles, on se rend compte que la saturation maximum n'est pas obtenue sur le périmètre du triangle en $\mathrm{A}$ mais en $\mathrm{A}^{\prime}$; ceci est vrai pour la plus grande partie du spectre. La courbe obtenue en joignant tous ces points correspondant à la saturation maximum est appelée la ligne spectrale.

Toutes les sensations colorées ne sont donc pas obtenues par l'addition pure et simple des trois primaires choisies.

On s'est efforcé de trouver un artifice pour annuler l'effet de cette anomalie.

.Pour cela, on a circonscrit la ligne spectrale dans un triangle rectangle isocèle. La déformation de la courbe ainsi produite n'affecte en rien la représentativité de l'aire des couleurs (fig. 3).

Dans le triangle, on détermine la chromaticité, c'est-à-dire la teinte et la saturation ; mais une couleur de chromaticité donnée peut être plus ou moins sombre.

Pour réellement représenter toutes les couleurs possibles, c'està-dire ajouter à la chromaticité la notion de brillance ou de luminance, il devient nécessaire de se servir d'un espace chromatique à trois dimensions.

Cet espace est représenté par un tétraèdre (fig. 4).

$\mathrm{Si}$, dans le plan de base figurant le triangle trichromatique, nous choisissons un point $\mathrm{A}$ quelconque, en traçant $\mathrm{AO}$, on joint dans le volume toutes les couleurs homologues, c'est-à-dire ayant la même chromaticité mais différant seulement par la brillance. En se déplaçant vers $\mathrm{O}$, la couleur s'assombrit de plus en plus et tend vers le noir.

Les coordonnées d'un point dans ce tétraèdre sont appelées coordonnées trichromatiques (XYZ) ; les coordonnées du même point sur le plan parallèle au triangle de base sont appelés coefficients trichromatiques (xyz). La correspondance entre coordonnées trichromatiques et coefficients trichromatiques est obtenue par les relations suivantes :

$$
\begin{aligned}
\frac{\mathrm{x}}{\mathrm{X}}=\frac{\mathrm{y}}{\mathrm{Y}} & =\frac{\mathrm{z}}{\mathrm{Z}} \text { et } \mathrm{x}+\mathrm{y}+\mathrm{z}=1 \\
\mathrm{x} & =\frac{\mathrm{X} \text { on a donc }:}{\mathrm{X}+\mathrm{Y}+\mathrm{Z}} \quad \mathrm{y}=\frac{\mathrm{Y}}{\mathrm{X}+\mathrm{Y}+\mathrm{Z}} \\
\mathrm{z} & =\frac{\mathrm{Z}}{\mathrm{X}+\mathrm{Y}+\mathrm{Z}}
\end{aligned}
$$




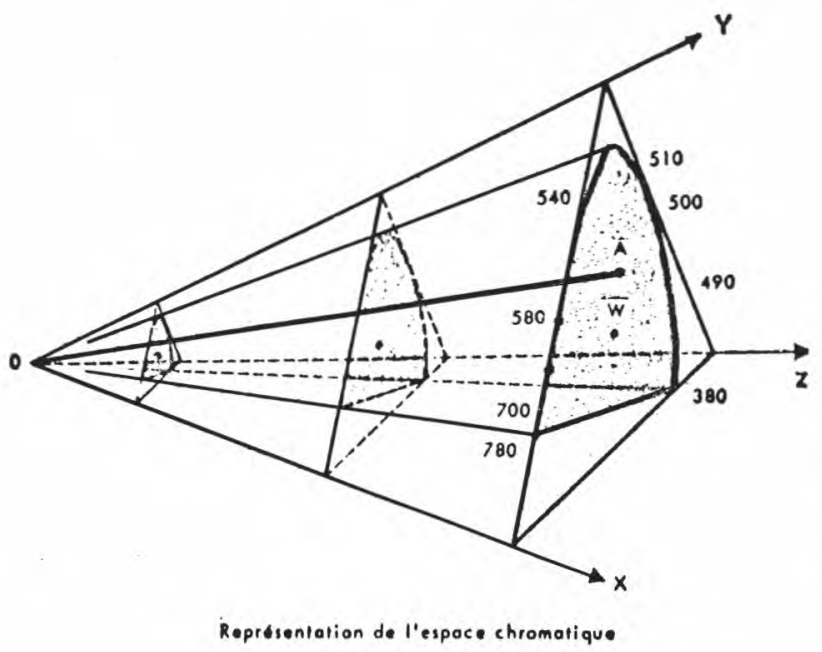

fig. 4

On peut donc bien, à partir des coordonnées $\mathrm{X}, \mathrm{Y}$ et $\mathrm{Z}$ définir exactement une couleur telle qu'elle est perçue pour l'œil. Les coordonnées $x$ et y permettent alors de déterminer, dans le triangle des couleurs, un point caractéristique de la chromaticité.

\section{II. - Méthodologie}

A) Mesures de la couleur

Appareillage : Les premiers travaux ont été menés avec un spectrophotomètre Jobin et Yvon, type Maroc, muni d'un dispositif pour la détermination, aux diverses longueurs d'onde, des coefficients de réflexion diffuse. En raison du temps nécessaire aux mesures, la température de l'échantillon variait fortement au cours de la lecture. De plus, la complexité et le nombre des calculs nécessitaient le recours à un ordinateur.

A la suite de ces observations, nous avons poursuivi nos travaux en mesurant directement la couleur au moyen d'un appareil "Zeiss Elrepho ". La mesure de trois paramètres Rx, Ry et Rz permet la détermination très rapide de $\mathrm{X}, \mathrm{Y}$ et $\mathrm{Z}$. Cet appareil, soumettant l'échantillon à un éclairage très violent, pouvant élever la température, nous avons procédé de la façon suivante :

- Tarage du témoin blanc à 100 pour le $1^{\text {er }}$ filtre ;

- Introduction de l'échantillon;

- Equilibrage du galvanomètre ;

- Introduction entre l'échantillon et la source d'un écran noir ; 
- Lecture de la mesure ;

- Tarage du témoin blanc à 100 pour le $2^{\text {me }}$ filtre ;

- Suppression de l'écran noir ;

- Equilibrage du galvanomètre.

Cette série d'opérations est effectuée avec le $3^{\text {me }}$ filtre puis reprise avec le $1^{\text {er }}$, le $2^{\text {me }}$ et le $3^{\text {me }}$. Si la différence entre les deux séries de mesures est inférieure à 2 p. 100, la précision est considérée comme suffisante, et l'on conserve les chiffres les plus élevés. En effet, lors d'une exposition un peu prolongée, l'échauffement provoque une modification de la couleur du beurre. Il est donc nécessaire d'opérer très rapidement pour que la température de l'échantillon ne varie pas trop entre le début et la fin des mesures.

Par ailleurs, en raison du type de support d'échantillon utilisé, il était nécessaire de protéger la surface de mesure et l'appareil contre les introductions de beurre. Ceci a été obtenu en intercalant entre l'échantillon et l'appareil une mince feuille de cellophane. Celle-ci entraîne les pertes suivantes :

- 2 p. 100 sur Rx.

- 2 p. 100 sur Ry.

- 3 p. 100 sur Rz.

Moyennant ces précautions, les résultats obtenus sont assez fidèles et reproductibles.

Préparation de l'échantillon : Pour obtenir des conditions homogènes entre les échantillons, les conditions de stockage ont été bien définies. Le beurre a été conservé au moins $24 \mathrm{~h}$ à $+5^{\circ} \mathrm{C}$, puis au moins $12 \mathrm{~h}$ à $+12^{\circ} \mathrm{C}$. Les porte-échantillons ont été garnis de beurre, recouverts de cellophane, puis maintenus environ $1 \mathrm{~h}$ à $+12^{\circ} \mathrm{C}$. Les mesures ont été faites selon le processus indiqué précédemment.

\section{B) Dosage Des PIgments}

La méthode utilisée au départ comportait trois phases :

Extraction : Saponification.

Purification : Chromatographie sur colonne d'alumine.

Mesure : Spectrophotométrie.

Après un certain nombre d'essais comparatifs, il a été constaté que l'on pouvait sans risque supprimer la chromatographie sur colonne d'alumine. La méthode s'en trouve simplifiée, ce qui permet de diminuer notablement le temps nécessaire à une détermination.

La saponification se fait à la potasse. La teneur en équivalent $\beta$-carotène est déterminée par l'extinction au maximum d'absorption $(448-450 \mu \mathrm{m})$ par rapport à un blanc éther de pétrole. Le spectre d'absorption est enregistré (spectrophotomètre-enregistreur Beck1 p. 100

man). Le $E \frac{1 \mathrm{~cm}}{1 u} \beta$-carotène au maximum d'absorption est de 2500 pour une solution dans l'éther de pétrole. 


\section{III. - Résultats}

A) Relation entre la couleur et la teneur en B-CARotène

\section{1) Nature des pigments du beurre}

La simplification de méthode décrite précédemment a été rendue possible, car elle avait peu d'incidences sur le dosage. L'examen des spectres d'absorption a permis par ailleurs de constater qu'ils correspondaient assez bien à celui du $\beta$-carotène. On peut donc considérer que ce corps est le pigment principal du beurre et que les résultats peuvent être, sans erreur importante, exprimés en $\beta$-carotène. En effet, si l'on se place sur le plan de la teneur en pigments, les autres caroténoïdes qui peuvent être présents, en faible quantité, ont des caractéristiques optiques voisines de ce produit. Aussi parlerons-nous simplement par la suite, de teneur en $\beta$-carotène.

La teneur en riboflavine n'a pas été prise en considération. En effet, le pouvoir pigmentant de ce produit est très faible par rapport aux caroténoïdes.

\section{2) Corrélation entre mesures trichromatiques et teneur en} $\beta$-carotène

Un certain nombre d'études ont déjà été effectuées. Elles ont, en général, mis en corrélation une mesure avec le classement visuel d'échantillons. Cette mesure, qu'elle soit relative à la couleur ou à la teneur en $\beta$-carotène, est en bonne corrélation avec l'examen visuel.

Lors de nos mesures de trichromie, il a été possible de comparer les teneurs en $\beta$-carotène aux coefficients trichromatiques. Pour 9 échantillons étudiés, 2 corrélations sont significatives à plus de 90 p. 100 :

$$
\begin{aligned}
& \mathrm{K}=1,2-0,016 \mathrm{Z} \\
& \mathrm{K}=9,16 \mathrm{y}-3,13
\end{aligned}
$$

$\mathrm{Z}=$ Coordonnée trichromatique.

$\mathrm{y}=$ Coefficient trichromatique.

$\mathrm{K}=$ Teneur $\mathrm{du}$ beurre en $\beta$-carotène.

Cette étude est poursuivie actuellement.

Les mesures de trichromie permettent donc, de façon simple, d'apprécier la couleur d'un beurre et d'estimer sa teneur en $\beta$-carotène. Elles permettent de constater que, dans des conditions bien déterminées, le seul facteur de variation de la couleur est la quantité de caroténoïdes contenus dans le beurre, exprimée en équivalents $\beta$-carotène.

\section{3) Autres facteurs influençant la couleur du beurre}

La couleur d'un produit solide dépend d'autres facteurs. Dans le cas du beurre, émulsion d'eau dans une phase grasse solide, plusieurs points sont à considérer. 
Ainsi, par exemple, la surface d'un beurre exposé à l'air jaunit lentement. Ceci est dû vraisemblablement à des phénomènes d'oxydation.

Sur toutes les émulsions, on constate des modifications de couleur liées à la finesse de la dispersion. Plus la dispersion est fine, plus la couleur de la phase continue est atténuée, et celle de la phase discontinue renforcée.

Enfin, le mode de cristallisation joue un rôle important dans la transmission de la lumière. Comme il dépend de la température de l'échantillon, il est important, avant tout examen, de maintenir pendant $24 \mathrm{~h}$, tous les échantillons à une température comprise entre 5 et $15^{\circ} \mathrm{C}$. Dans notre étude, les déterminations ont été faites sur des beurres tempérés à $12^{\circ} \mathrm{C}$ en début de mesure.

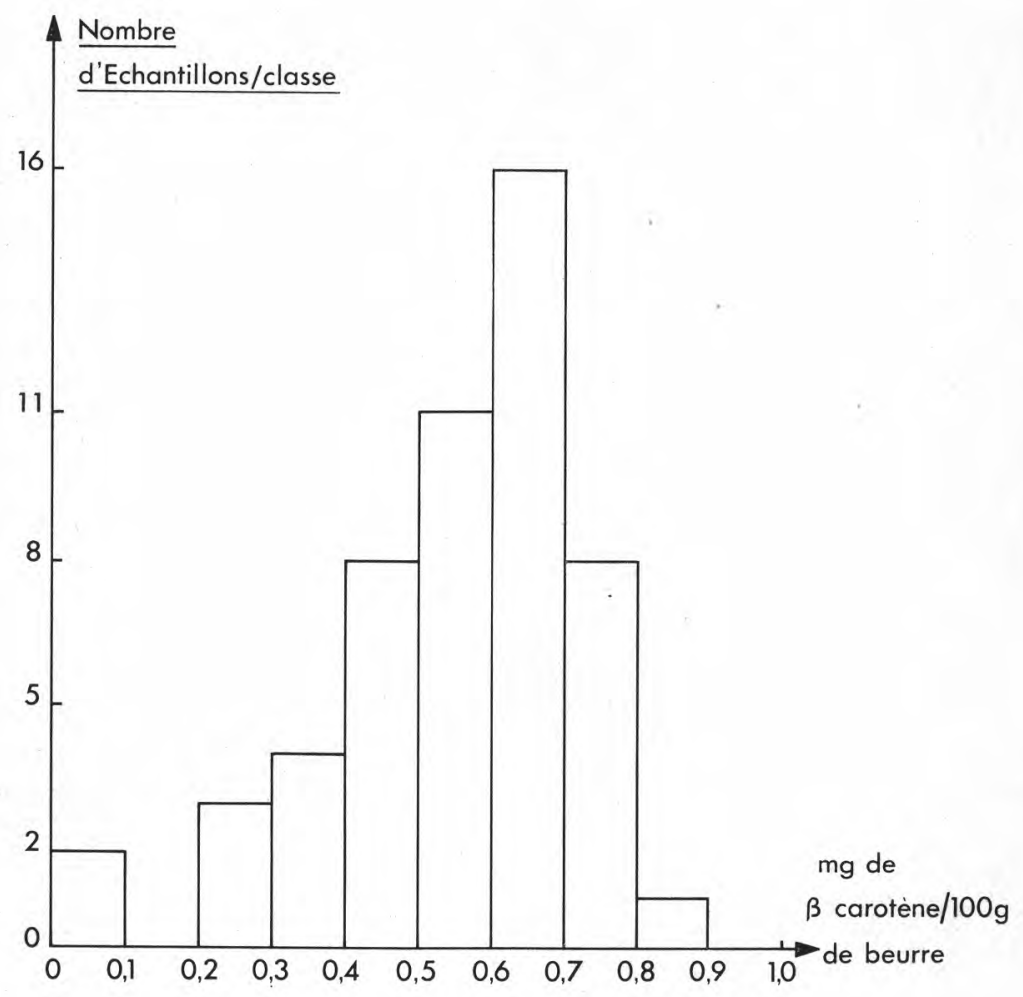

fig. 5

Répartition par classe des teneurs en $\beta$-carotène des échantillons de beurre sur le marché parisien 
B) ENQUÊTES

1) Teneur en pigments d'échantillons de beurres prélevés sur le marché parisien

Dans un premier temps, nous avons rassemblé un certain nombre de résultats obtenus lors d'une enquête alimentaire. Celle-ci portait sur 53 beurres de toutes origines, achetés sur le marché parisien en 1969-1970. La figure 5 indique les répartitions par classe de teneur en $\beta$-carotène de ces échantillons. On peut en dégager les faits suivants :

- Il existe, sur le marché, des beurres dont la teneur en $\beta$-carotène est voisine de 0 .

- Près de 70 p. 100 des échantillons prélevés ont une teneur comprise entre 0,5 et $0,8 \mathrm{mg}$ de $\beta$-carotène pour $100 \mathrm{~g}$ de beurre, la classe 0,6-0,7 représentant à elle seule plus de 30 p. 100 du total.

\section{2) Etude des variations saisonnières}

Les variations saisonnières de coloration ont été étudiées sur des échantillons de beurre prélevés chaque semaine (du 27 février au 3 juillet 1970) dans une même exploitation agricole de Normandie, où l'on n'ajoute pas de colorant aux crèmes. Les résultats des analyses de ces échantillons sont consignés au tableau de la figure 6 .

\begin{tabular}{c|c|c|c|c|c|c|c|c|c}
\hline Dates & $27 / 2$ & $6 / 3$ & $13 / 3$ & $20 / 3$ & $27 / 3$ & $3 / 4$ & $10 / 4$ & $17 / 4$ & $24 / 4$ \\
\hline Teneur en $\beta$-carotène & 0,340 & 0,435 & 0,400 & 0,425 & 0,435 & 0,540 & 0,605 & 0,660 & 0,745 \\
\hline
\end{tabular}

\begin{tabular}{c|c|c|c|c|c|c|c|c|c}
\hline $1 / 5$ & $8 / 5$ & $15 / 5$ & $22 / 5$ & $29 / 5$ & $5 / 6$ & $12 / 6$ & $19 / 6$ & $26 / 6$ & $3 / 7$ \\
\hline 0,725 & 0,690 & 0,675 & 0,580 & 0,585 & 0,715 & 0,595 & 0,565 & 0,490 & 0,475 \\
\hline
\end{tabular}

fig. 6

Teneur en $\beta$-carotène de beurres fermiers normands

La figure 6 fait apparaître deux teneurs maxima en $\beta$-carotène : l'une fin avril, et l'autre début juin. Ceci semble correspondre à la repousse de l'herbe et à la floraison. En l'espace de 4 mois, l'hétérogénéité de teneur en pigments est très grande (valeurs extrêmes 0,340 et $0,745 \mathrm{mg} / 100 \mathrm{~g}$ beurre). D'autre part les variations sont très soudaines. Pendant le mois d'avril les teneurs en $\beta$-carotène passent de 0,435 à $0,745 \mathrm{mg} / 100 \mathrm{~g}$ de beurre. 


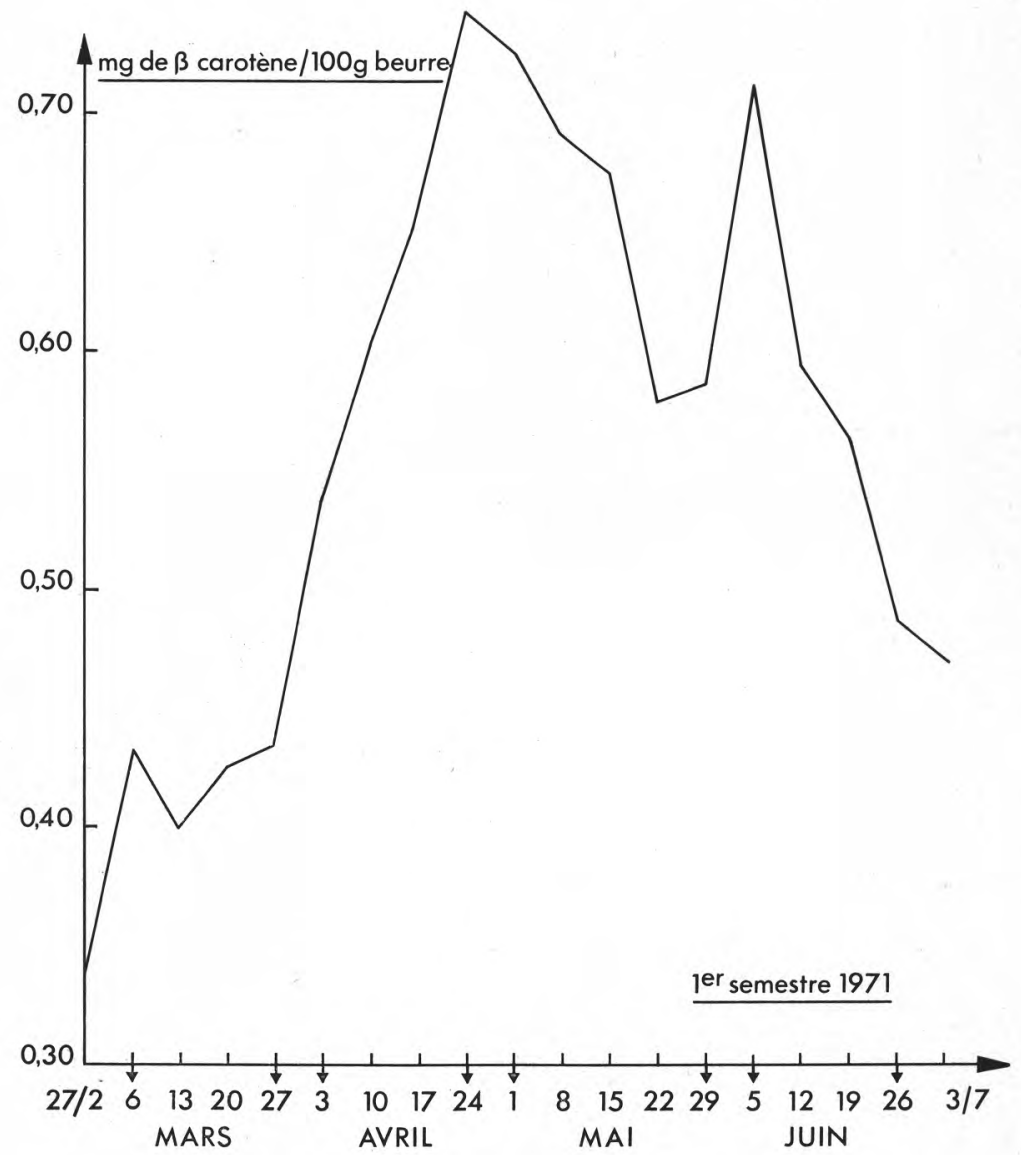

fig. 7

Variations de la teneur en $\beta$-carotène d'un même beurre fermier pendant le premier semestre 1971

3) Enquête sur des beurres de l'Est et de l'Ouest de la France

Chaque mois ont été examinés des échantillons de beurre provenant de différentes entreprises françaises de l'Est et de l'Ouest. Tous ces beurres avaient été fabriqués sans adjonction de colorant. Les résultats des dosages de $\beta$-carotène sont regroupés dans le tableau de la figure 8 et représentés dans le graphique de la figure 9 . 


\begin{tabular}{|c|c|c|c|c|c|c|}
\hline Mois & Janvier & Février & Mars & Avril & Mai & Juin \\
\hline Beurres Est & 0,180 & 0,140 & 0,150 & 0,310 & 0,480 & 0,560 \\
\hline Beurres Ouest & - & 0,440 & 0,520 & 0,670 & 0,710 & 0,620 \\
\hline Mois & Juillet & Août & Sept. & Octobre & Nov. & Déc. \\
\hline Beurres Est & 0,500 & 0,390 & 0,420 & 0,490 & 0,360 & 0,320 \\
\hline Beurres Ouest & 0,600 & 0,530 & 0,580 & 0,600 & 0,565 & - \\
\hline
\end{tabular}

Teneur en $\beta$-carotène des beurres des régions Est et Ouest (année 1971)

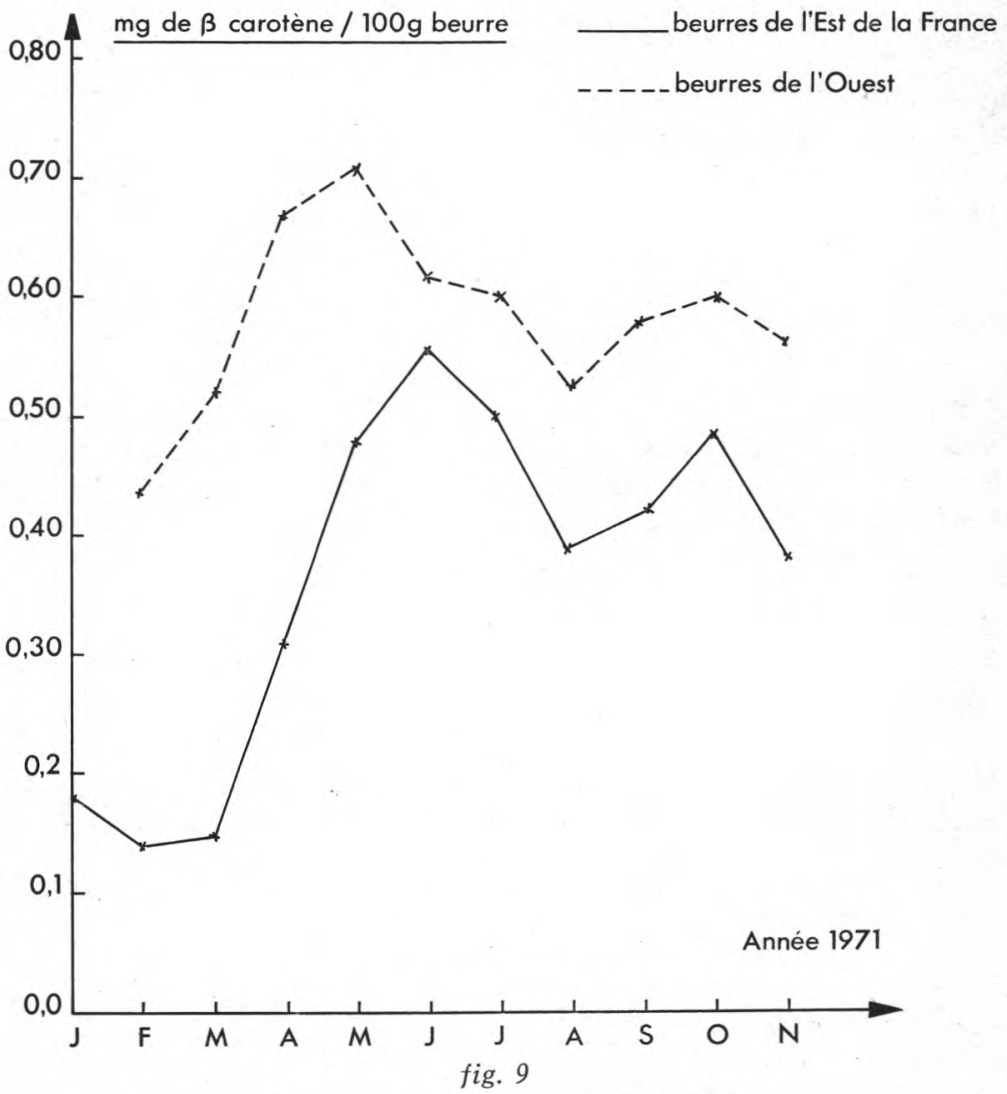

Variations annuelles de la teneur en $\beta$-carotène 
Ces résultats sont comparables à ceux obtenus par Hugot et Causeret, et permettent de dégager les conclusions suivantes :

- les teneurs en $\beta$-carotène sont toujours plus fortes dans les beurres de l'Ouest (dès le mois de mars, la teneur en $\beta$-carotène reste supérieure à $0,5 \mathrm{mg} / 100 \mathrm{~g}$ de beurre);

- la remontée des teneurs en $\beta$-carotène au printemps est plus tardive pour les beurres de l'Est (exemple : en avril respectivement $0,310 \mathrm{mg}$ (Est) et $0,670 \mathrm{mg}$ (Ouest) pour $100 \mathrm{~g}$ de beurre ;

- les teneurs maxima sont légèrement décalées entre les deux groupes : mai pour les beurres Ouest, et juin pour les beurres Est;

- après un fléchissement en août, une remontée des teneurs pendant les mois d'automne est probablement due à l'alimentation du bétail, et à une dernière repousse de l'herbe.

\section{Conclusion}

La teneur moyenne en $\beta$-carotène des beurres vendus sur le marché français est de l'ordre de 6,5 ppm.

Ses variations saisonnières et ses variations régionales sont fort importantes.

Les régimes alimentaires du bétail, qui correspondent à des régions différentes, peuvent entraîner d'autres variations.

Il est certain que nous vivons à une époque où les échanges s'intensifient, où le stockage sur de longues périodes est chose facile à réaliser, où les circuits de distribution sont de plus en plus fluctuants. Dans cette conjoncture, la possibilité de normaliser la couleur du beurre permet de mieux adapter la production à la demande du consommateur.

La demande, en France, concerne surtout les beurres de couleur assez intense. La méthode de mesure de la coloration du beurre dont nous proposons de poursuivre l'étude, et qui permettrait de déterminer les quantités exactes de pigment à ajouter à la crème, faciliterait singulièrement la réalisation de la normalisation souhaitable.

\section{Remerciements}

Nous remercions très sincèrement les entreprises laitières qui nous ont gracieusement envoyé les échantillons du beurre nécessaires à nos recherches.

\section{Résumé}

La couleur du beurre est, en France un important facteur extérieur de qualité. Aussi a-t-on recherché les éléments qui en sont res- 
ponsables. Quelques rappels de colorimétrie permettent de mieux définir l'appréciation objective d'une couleur.

Les déterminations trichromatiques ont été faites à l'aide d'un "Zeiss Elrepho ». Le dosage du $\beta$-carotène a pu être simplifié dans le cadre de cette étude. Les résultats ont permis de mettre en évidence :

- le rôle joué par le $\beta$-carotène comme pigment du beurre ;

- les relations existant entre détermination trichromatique et taux de $\beta$-carotène dans les échantillons.

Plusieurs enquêtes effectuées montrent bien les variations régionales et saisonnières du taux de $\beta$-carotène des beurres. Des séries de prélèvements au niveau du consommateur indiquent une préférence pour les beurres contenant de 6 à $7 \mathrm{ppm}$ de $\beta$-carotène.

La méthode proposée de mesure de la coloration du beurre permet de déterminer les quantités exactes de $\beta$-carotène à ajouter à la crème pour réaliser la normalisation de la couleur du beurre.

\section{S u m m a ry}

In France, the colour of butter is an important factor of quality. So have we looked for its responsible factors. Some recalls of colorimetry let better determine an objective estimation of colour.

Trichromatic determinations were made with a "Zeiss EIrepho ". Assays have allowed a simplification of $\beta$-carotene determination. The results have shown :

- The role played by $\beta$-carotene as butter pigment;

- The relations between trichromatic data and amount of $\beta$-carotene in the different samples.

Surveys, made during the same time, show important variations due to seasons and areas. Some serial sampling at consumer's home indicate a preference for butters containing 6 to $7 \mathrm{ppm}$ of $\beta$-carotene.

The purposed method of color measurement allows the exact determination of added $\beta$-carotene in cream to obtain the standardisation of butter colour.

\section{Bibliographie}

ANONYME. - Investigation of various methods for determining the colour of butter. 166 beretning. Statens Fors $\varnothing$ gsmejeri-Hillerød (Danemark).

Bouma (P. J.) (1949). - Les couleurs et leur perception visuelle. Ed. NV Philips Gloeilampenfabrieken, Eindhoven, Pays-Bas.

Byers (J. H.) and al. (1955). - Carotene in the ration of dairy cattle. J. of Dairy Science, 38, 657-663. 
GAzo (M.). (1958). - Metabolism of Vitamin A an Carotene. II/In dairy cows, in relation to season and nutrition. Veterinarsky Casopis, 7, $\mathrm{n}^{\circ} 1$, 66-79.

Goodwin (T.W.) (1952). - The comparative Biochemistry of the Carotenoids. Ed. Chapman et Hall Ltd, Londres.

Hugot (D.) et CAuseret (J.) (1966). - Variations saisonnières de l'activité vitaminique A de beurres produits dans diverses régions de France. $17^{\mathrm{e}}$ Congrès Int. Laiterie, C 185-C 191.

JUDD (D. B.) (1961). - Color in business, Science and industry. $3^{\text {me }}$ édition, John Wiley et Sons, Inc., New-York.

KARRER (P.) and JUCKER (E.) (1950). - Carotenoids. Elsevier Publishing Compagny Inc.

LücK (H.) and Novello (J.) (1970). - Measuring and Standardising the colour of butter. South African Journal Dairy Tech., 2, $\mathrm{n}^{\circ}$ 2, 91-96.

Mac GillivraY (W.A.) and Thompson (S.Y.) (1957). - The influence of pasture on the Vitamin A and Carotene in the milk of cows. Proceed. of the Nutr. Soc., $16,30-36$.

Mackinney (G.) and Litrle (A. C.) (1962). - Color of Foods. The AVI Publishing Compagny Inc., Westport, Connecticut.

Mainguy (P.) et Rouques (A.) (1963). - La couleur vitelline. F. HoffmannLa Roche, Paris.

Searles (S. K.) and Armstrong (J. G.) (1970). - Vitamin A, vitamin E and Carotene contents of Alberta butter. J. of Dairy Science, 53, $\mathrm{n}^{\circ}$ 2, 150-154.

Soll Berl and Peterson (W. H.) (1943). - Determination and content of Carotene and vitamin $\mathrm{A}$ in Wisconsin Butter. J. of Nutrition, 26, $\mathrm{n}^{\circ} 1,527-538$.

Thompson (S. Y.) (1959). - Further Studies of factors influencing the carotenoids and vitamin A content of milk in Great Britain. Proceedings -

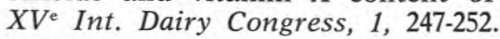

Wright (W. D.) (1958). - The Measurement of colour. $2^{\text {me }}$ édition, Hilger et Watts Ltd, Londres.

YVER (M.) et MAINGUY (P.) (1971). - Les caroténoïdes, nouveaux agents colorants mis à la disposition des industries alimentaires. Cycle conférences $C T U$, certains additifs chimiques, 31-41. 